\title{
Dynamic charging using parallel line feeder with hybrid inductive-capacitive coupling and receiver-side load control
}

\author{
Quang-Thang Duonga), Takuya Maekawa, \\ Takeshi Higashino, and Minoru Okada \\ Graduate School of Information Science, Nara Institute of Science and Technology, \\ 8916-5 Takayama-cho, Ikoma, Nara 630-0192, Japan
}

a) thang@is.naist.jp

\begin{abstract}
This paper proposes a dynamic charging system using hybrid inductively-capacitively coupled parallel line feeder with receiver-side load control to guarantee a stable target power profile for mobile receiver. Inductive- or capacitive-coupling system suffers from nulls in power profile due to impact of standing waves when operating in the HF band. Because current and voltage standing waves are out of phase by 90 degrees, hybrid inductive-capacitive coupling is employed to remove nulls in the power profile. Furthermore, load is iteratively controlled at the receiver until reaching the target power. Analysis and experimental results show that the load control is notification-free and fast converging compared to ordinary vehicle speed.
\end{abstract}

Keywords: dynamic charging, parallel line feeder, standing wave, hybrid inductive-capacitive coupling, receiver-side load control

Classification: Electronic instrumentation and control

\section{References}

[1] G. A. Covic and J. T. Boys: Proc. IEEE 101 (2013) 1276. DOI:10.1109/ JPROC.2013.2244536

[2] T.-E. Stamati and P. Bauer: IEEE Transp. Electrific. Conf. and Expo. (2013) 1. DOI:10.1109/ITEC.2013.6573511

[3] O. C. Onar, J. M. Milner, S. L. Campell, C. Coomer, C. P. White and L. E. Seiber: IEEE Appl. Power Electron. Conf. and Expo. (2013) 3073. DOI:10. 1109/APEC.2013.6520738

[4] J. T. Boys, G. A. Covic and A. W. Green: IEE Proc. Electric Power Appl. 147 (2000) 37. DOI:10.1049/ip-epa:20000017

[5] J. Shin, B. Song, S. Lee, S. Shin, Y. Kim, G. Jung and S. Jeon: IEEE Int. Electric Vehicle Conf. (2012) 1. DOI:10.1109/IEVC.2012.6183255

[6] T. Higashino, M. Ziji, M. Okada, Y. Tatsuta, Y. Goto, Y. Tsuruda and R. Tanaka: IEEE Wireless Power Transfer Conf. (2013) 171. DOI:10.1109/WPT. 2013.6556910 
[7] T. Ohira: IEEE Wireless Power Transfer Conf. (2013) 242. DOI:10.1109/WPT. 2013.6556928

[8] D. M. Pozar: Microwave Engineering (Wiley, New York, 2012) 4th ed. 48.

[9] T. Ishizaki, G. Kitano and K. Mikami: IEEE Wireless Power Transfer Conf. (2014) 142. DOI:10.1109/WPT.2014.6839575

[10] W. H. Press, B. P. Flannery, S. A. Teukolsky and W. T. Vetterling: Numerical Recipes in FORTRAN (Cambridge University Press, Cambridge, 1992) 2nd ed. 347.

\section{Introduction}

Recently, dynamic charging [1,2] for automated guided vehicles (AGVs) and future electric vehicles (EVs) has attracted great attention in both academia and industry. A typical dynamic charging system consists of a power feeder buried under the roadway and couplers mounted on mobile receivers. While being on the move, the receivers inductively and/or capacitively couple power from electromagnetic field generated by the feeder.

Several feeder structures have been proposed to support receiver mobility. In [3], many small feeding coils are placed one next to each other, allowing one feeding coil to charge the receiver at every moment of time. This structure is highly efficient but complex and costly, because it requires an inverter for powering each coil [2], as well as a fast vehicle sensing to quickly tune the coil, on which the vehicle is passing-by. Systems proposed in [4] and [5] use one elongated feeder to charge the receiver moving within the large feeding area. These systems have lower efficiencies because the receiver picks up only one portion of the electromagnetic field. However, considering their simplicity and low cost, systems using one elongated feeder are promising options for practical dynamic charging if their efficiencies are improved.

Operating frequency is then raised from below $300 \mathrm{kHz}$ as in $[3,4,5]$ to within the high frequency (HF: 3-30 MHz) band to make further improvements. In [6], for further reduction of system size and cost, an HF generator is used to power an inductively-coupled parallel line feeder. Because HF displacement currents pass through asphalt and tires, capacitive-coupling is exploited in [7] to realize zero-gap power transfer from the feeder to steel belts of vehicle wheels. However, because the wavelength of HF signal $(10-100 \mathrm{~m})$ is shorter than the feeder length (several tens meters to kilometers), current and voltage along the feeder follow standing wave pattern which is an alternative of nodes and anti-nodes [8]. As a consequence, both systems in [6] and [7] suffer from nulls in power profile, forcing the receiver to consume battery.

Battery accounts for about $60 \%$ of an EV cost. In order to avoid battery usage, guaranteeing a target power profile is of utmost importance. Impact of standing waves can be mitigated if the feeder is terminated in its characteristic impedance [8]. However, in such a case, the terminating impedance consumes most power from the source since it is matched to the feeder. Another method is to use directional coupler and filter as in [9], but this method is, unfortunately, applicable 


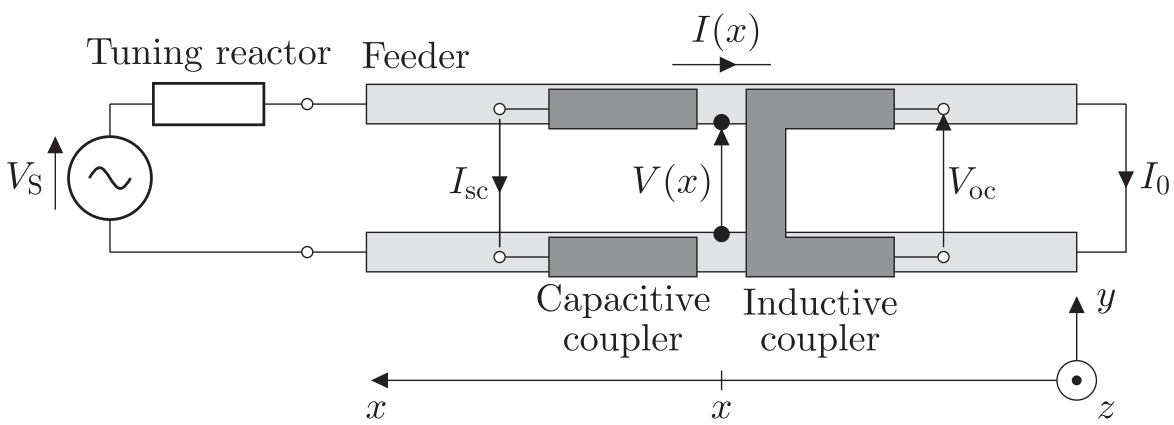

Fig. 1. Dynamic charging using parallel line feeder.

to microwave systems, which are subject to severe radiation loss. Hence, guaranteeing a stable target power profile is still an open issue. To resolve this issue, this paper proposes a dynamic charging system using hybrid inductively-capacitively coupled parallel line feeder with receiver-side load control. Our contributions are twofold.

- Removing nulls in the power profile by employing hybrid inductive-capacitive coupling to draw power from current and/or voltage standing waves which are out of phase by 90 degrees.

- A notification-free load control which iteratively adjusts load impedance at the receiver until reaching the target power.

Experimental results show that the load control is fast converging compared to ordinary vehicle speed, verifying effectiveness of the proposed system.

The rest of the paper is organized as follows. Section 2 introduces dynamic charging using parallel line feeder. Hybrid inductive-capacitive coupling is presented in section 3. Receiver-side load control is given in section 4 and conclusions are drawn in the last section.

\section{Dynamic charging using parallel line feeder}

\subsection{System model}

Dynamic charging system using parallel line feeder is shown in Fig. 1. At the transmitter, a sinusoidal HF generator with voltage amplitude $V_{\mathrm{S}}$ and operating frequency $f$ is used as the power source. The feeder consists of two identical wires placed parallel to each other. The feeder is connected to the source via a tuning reactor to compensate for its reactance and terminated at the other end in a short circuit. Characteristic impedance of the feeder is $Z_{0}$. We assume a receiver is moving on top of the feeder and along the $x$-axis. Position of the receiver is denoted by its distance $x$ to the terminating end.

An incident wave generated from the source will be reflected at the terminating end $(x=0)$ and then combined with another incident wave to form a standing wave along the feeder. According to transmission line theory [8], current standing wave is characterized by

$$
I(x)=I_{0} \cos \frac{2 \pi x}{\lambda},
$$

where $I(x)$ is current amplitude at $x, I_{0}$ is current amplitude at $x=0$ and $\lambda$ is the wavelength. Similarly, voltage standing wave is characterized by 


$$
V(x)=j Z_{0} I_{0} \sin \frac{2 \pi x}{\lambda},
$$

where $V(x)$ is voltage amplitude between the two parallel lines and $j=\sqrt{-1}$.

\subsection{Standing wave issue}

If the receiver uses an inductive-coupler, e.g., a loop coil, to pick up power from the current $I(x)$, the inductive-coupler can be viewed as a voltage source whose opencircuit voltage

$$
V_{\mathrm{oc}}(x)=j 2 \pi f L_{\mathrm{m}} I(x)=j 2 \pi f L_{\mathrm{m}} I_{0} \cos \frac{2 \pi x}{\lambda} .
$$

In Eq. (3), $L_{\mathrm{m}}$ is mutual inductance between the feeder and the coupler. The available power that can be drawn from the inductive-coupler is dominated by the open-circuit voltage $V_{\mathrm{oc}}(x)$. It is shown in Eq. (3) that when $x$ varies, $V_{\mathrm{oc}}(x)$ fluctuates in a harmonic manner. As a result, the receiver suffers from a fluctuation in received power when moving along the feeder. More importantly, $V_{\mathrm{oc}}(x)=0$ at $x=(2 k+1) \lambda / 4$, where $k \in\{0,1,2, \ldots\}$. These positions are current nodes, where the receiver is not able to inductively pick up power, regardless of source voltage and load impedance.

Reversely, if the receiver uses a capacitive-coupler, e.g., two electrodes, to pick up power from the voltage $V(x)$, the coupler can be seen as a current source whose short-circuit current is

$$
I_{\mathrm{sc}}(x)=\frac{1}{j 2 \pi f C_{\mathrm{m}}} V(x)=\frac{Z_{0} I_{0}}{2 \pi f C_{\mathrm{m}}} \sin \frac{2 \pi x}{\lambda} .
$$

In Eq. (4), $C_{\mathrm{m}}$ denotes mutual capacitance between the capacitive-coupler and the feeder. Similar to the inductive-coupler case, $I_{\mathrm{sc}}(x)$ dominates available power that can be drawn from the capacitive-coupler. Eq. (4) indicates that in the case of capacitive-coupling, the receiver also suffers from a fluctuation in received power while moving along the feeder. Moreover, the receiver is not able to capacitively couple power at voltage nodes given by $x=k \lambda / 2$.

While moving along the $x$-axis, the receiver requires a stable target power for its operations. However, as mentioned above, if inductive- or capacitive-coupling is employed, there are positions where the receiver is not able to pick up power, regardless of source voltage and load impedance. These positions are nulls in power profile, which need to be coped with to guarantee a target power $P_{0}$ for the receiver. To this end, we first employ a hybrid inductive-capacitive coupling to remove nulls in the power profile. Then, load impedance is iteratively adjusted at the receiver until reaching a vicinity of the target value $P_{0}$. Such a load control is practical because its does not require any kind of notification between transmitter and receiver.

\section{Hybrid inductive-capacitive coupling}

\subsection{Receiver structure}

Eq. (4) indicates that $I_{\mathrm{sc}}(x)$ is maximum at current nodes $(x=(2 k+1) \lambda / 4)$. In such positions, although power is not available in the inductive-coupler, it can be drawn 
Table I. Experiment parameters.

\begin{tabular}{ll}
\hline$f$ & $13.56 \mathrm{MHz}$ \\
\hline$V_{\mathrm{S}}$ & $5 \mathrm{~V}$ \\
\hline Wire of feeder and couplers & Copper tape: $0.035 \mathrm{~mm} \times 10 \mathrm{~mm}$ \\
\hline Feeder (Two parallel wires) & Length: $17 \mathrm{~m}$, Width: $50 \mathrm{~mm}$ \\
\hline Inductive-coupler (U-shape) & Length: $100 \mathrm{~mm}$, Width: $50 \mathrm{~mm}$ \\
\hline Capacitive-coupler (Two parallel wires) & Length: $100 \mathrm{~mm}$, Width: $50 \mathrm{~mm}$ \\
\hline \multirow{2}{*}{ Air gap } & To inductive-coupler: $4 \mathrm{~mm}^{\mathrm{i}}$ \\
\hline & To capacitive-coupler: $1 \mathrm{~mm}^{\mathrm{i}}$ \\
Tuning & For feeder: $200 \mathrm{pF}$ \\
& For inductive-coupler: $1 \mathrm{nF}$ \\
\hline \multirow{2}{*}{ Rectifier } & For capacitive-coupler: $5 \mu \mathrm{H}$ \\
\hline
\end{tabular}

${ }^{\mathrm{i}}$ In this paper, air gaps are set to small values to facilitate capacitive and inductive couplings for experimental purpose. In practice, reasonable values for the air gaps, e.g., several centimeters and larger values can be achieved by scaling up the system and applying air gap enhancing methods.

Feeder

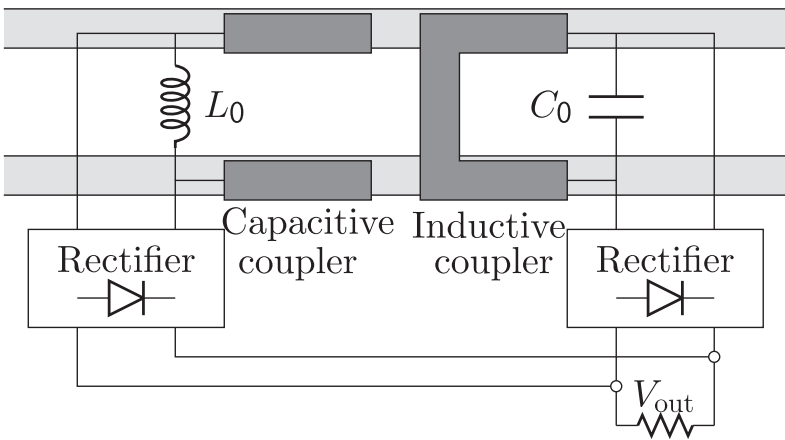

Load $R$

Fig. 2. Hybrid inductive-capacitive coupling.

from the capacitive-coupler. Reversely, at voltage nodes $(x=k \lambda / 2)$, although power is not available in the capacitive-coupler, it can be drawn from the inductive-coupler because $V_{\mathrm{oc}}(x)$ is maximum as shown in Eq. (3). Therefore, we employ a hybrid inductive-capacitive coupling to remove nulls in the power profile.

The hybrid inductive-capacitive coupling is illustrated in Fig. 2. The two couplers are parallel tuned by inductor $L_{0}$ and capacitor $C_{0}$, respectively. Outputs of the couplers are separately rectified to direct currents before connected in parallel to a single load $R$. Because the load $R$ receives power from the current $I(x)$ and/or the voltage $V(x)$, which are out of phase by 90 degrees, the hybrid coupling is promising to remove nulls in power profile.

\subsection{Power profile}

An experiment is carried out to investigate the power profile of the hybrid inductive-capacitive coupling. In this experiment, we built a simple system describ- 


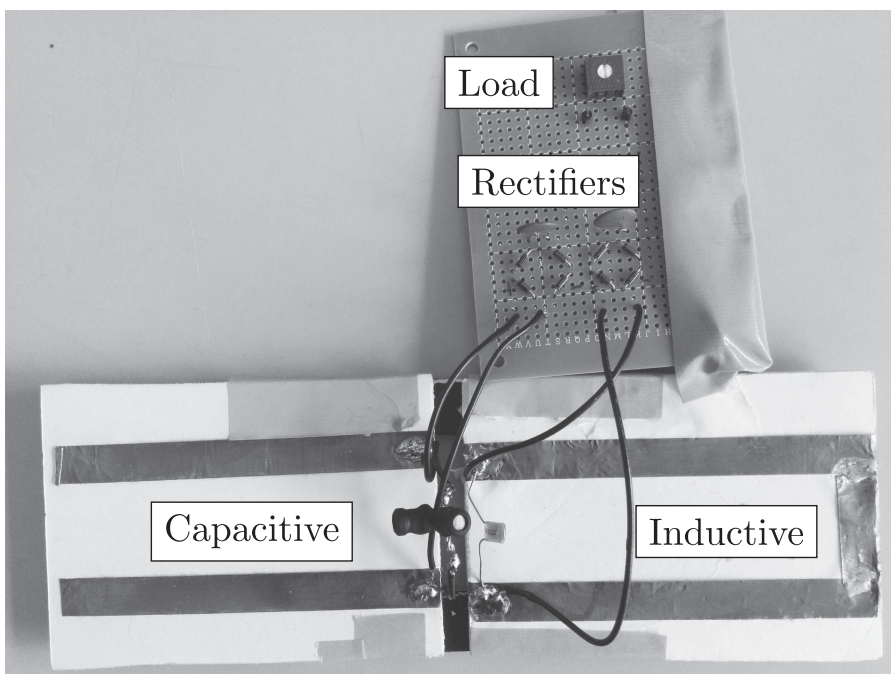

Fig. 3. A model of hybrid inductive-capacitive coupler.

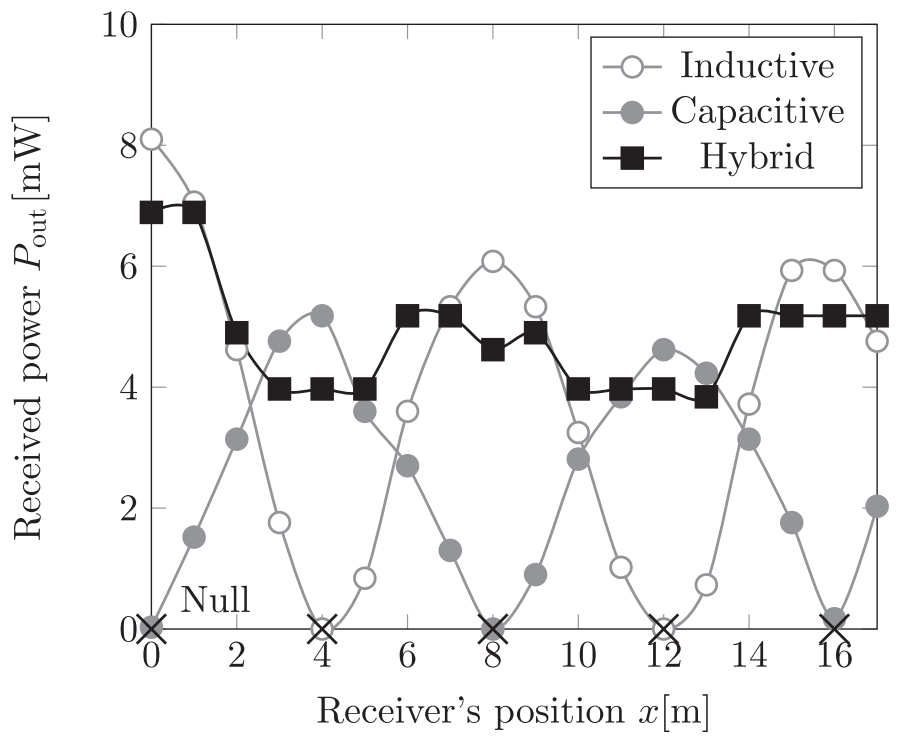

Fig. 4. Power profiles of three coupling schemes with $R=100 \Omega$.

ed in Figs. 1 and 2 with parameters given in Table I. A model of the proposed hybrid coupler is shown in Fig. 3. Received power is calculated by $P_{\text {out }}=V_{\text {out }}^{2} / R$, where $V_{\text {out }}$ is load voltage.

Fig. 4 demonstrates power profiles of three coupling schemes: inductivecoupling, capacitive-coupling and the proposed hybrid inductive-capacitive coupling. The inductive-coupling curve exhibits a harmonic variation with receiver's position with nulls at $x=4 \mathrm{~m}, 12 \mathrm{~m}$ where $P_{\text {out }}$ is almost $0 \mathrm{~mW}$. The capacitivecoupling curve shows a similar harmonic variation but with nulls shifted by $4 \mathrm{~m}$ to locations $x=0 \mathrm{~m}, 8 \mathrm{~m}, 16 \mathrm{~m}$. Meanwhile, the minimum received power of the hybrid coupling curve is as high as about $4 \mathrm{~mW}$. The hybrid coupling does not result any nulls in its power profile although a variation in received power is observed. The reason is that the scheme draws energy from $I(x)$ and/or $V(x)$, which are out of phase by 90 degrees as mentioned in section 3.1. Furthermore, Fig. 4 also indicates that wavelength $\lambda=16 \mathrm{~m}$ for the experimental system. 


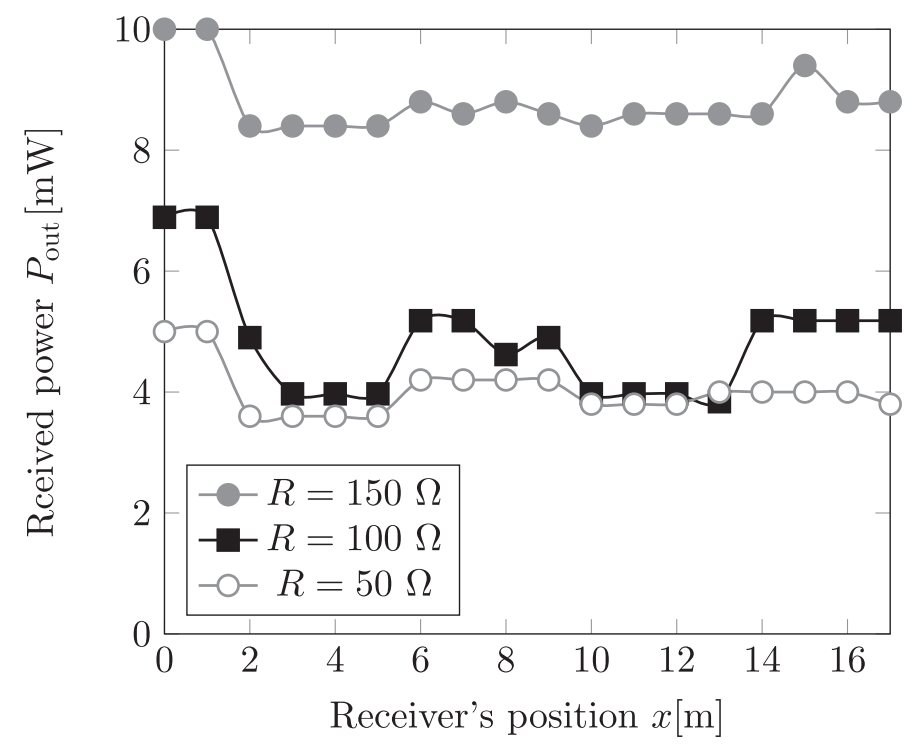

Fig. 5. Power profile of hybrid inductive-capacitive coupling.

Fig. 5 describes power profile of the hybrid coupling scheme for $R=50,100$, $150 \Omega$. The figure confirms the effectiveness of the hybrid coupling in removing nulls even under various values of load $R$. The figure also implies that the received power varies when load changes, suggesting the idea of load control for guaranteeing a target power profile.

\section{Receiver-side load control}

\subsection{Procedure}

In this section, load $R$ is controlled at the receiver to guarantee that received power is in a vicinity of a target power $P_{0}$. While moving, the receiver periodically measures its received power $P_{\text {out }}=V_{\text {out }}^{2} / R$ and changes the load if $P_{\text {out }}$ is out of the range $\left[(1-\varepsilon) P_{0},(1+\varepsilon) P_{0}\right]$, where $\varepsilon$ is a tolerance. The receiver gradually adjusts its load until $P_{\text {out }}$ falls in the range $\left[(1-\varepsilon) P_{0},(1+\varepsilon) P_{0}\right]$. The proposed load control is illustrated in Fig. 6 including steps below.

- Step 0: Set two initial values $R^{(0)}$ and $R^{(1)}$. Set iteration counter $n:=1$.

- Step 1: Measure $P_{\text {out }}^{(n)}$.

- Step 2: Compare $P_{\text {out }}^{(n)}$ and $P_{0}$. If $P_{\text {out }}^{(n)} \in\left[(1-\varepsilon) P_{0},(1+\varepsilon) P_{0}\right]$ then output $n$ and $R^{(n)}$ and stop this process. Else, go to step 3 .

- Step 3: Increase iteration counter $n$. Update $R^{(n)}$ by using secant method [10]. The secant method is used because of its fast convergence and simplicity.

$$
R^{(n)}=R^{(n-1)}-\left[R^{(n-1)}-R^{(n-2)}\right] \frac{P_{\text {out }}^{(n-1)}-P_{0}}{P_{\text {out }}^{(n-1)}-P_{\text {out }}^{(n-2)}} .
$$

After updating $R^{(n)}$ go to step 1 .

Fig. 7 describes an example of load control when the receiver is at position $x=1 \mathrm{~m}$. In this case, the initial values of load are $R^{(0)}=0 \Omega, R^{(1)}=50 \Omega$. Target power is $P_{0}=2.0 \mathrm{~mW}$ with tolerance $\varepsilon=0.1$. The figure shows the variation of load $R$ and the received power $P_{\text {out }}$ through iteration counter $n$. The load $R$ is 


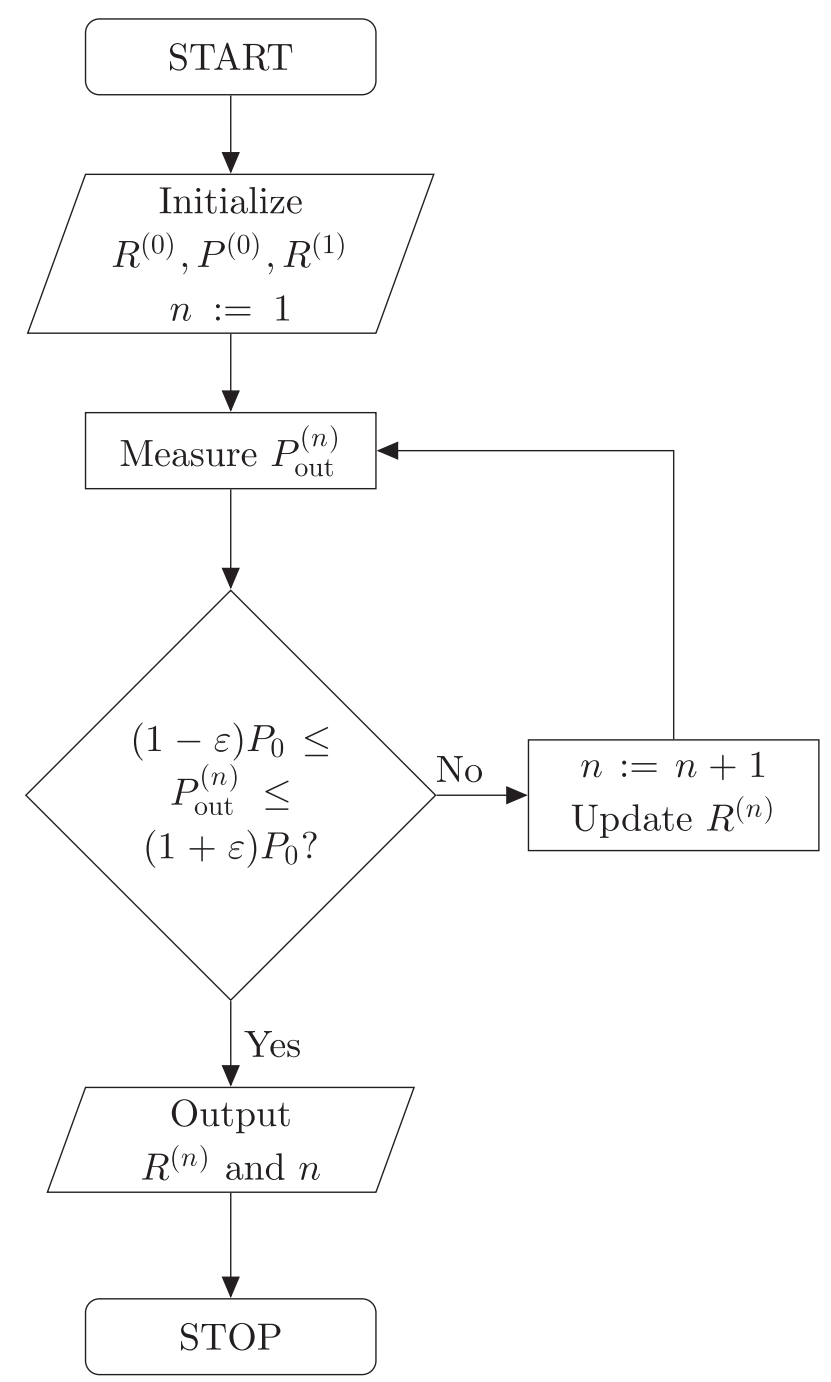

Fig. 6. Receiver-side load control procedure.

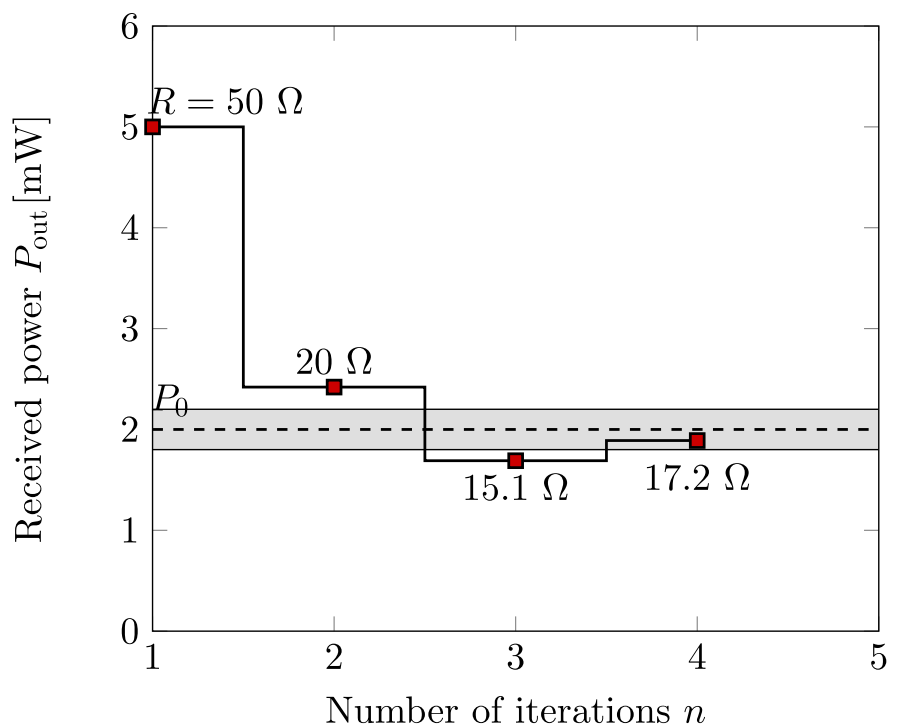

๑) IEICE 2015

DOI: $10.1587 /$ elex.12.20150854 Received October 9, 2015 Accepted October 26, 2015

Publicized November 9, 2015

Copyedited December 10, 2015 
gradually varied from the initial value $50 \Omega$, to $20.0 \Omega$ and $15.1 \Omega$. Finally, the load reaches $17.2 \Omega$ yielding a received power $P_{\text {out }}=1.89 \mathrm{~mW}$, which falls into the range $[1.8,2.2]$. The process ends after 4 iterations.

\subsection{Received power}

Fig. 8 plots power profile when receiver-side load control is applied to the system of section 3.2. Target power $P_{0}$ is set to $2 \mathrm{~mW}$ and $4 \mathrm{~mW}$ with tolerance $\varepsilon=0.1$. As shown in the figure, although slightly varies with position $x$, the received power totally falls into the target range $\left[(1-\varepsilon) P_{0},(1+\varepsilon) P_{0}\right]$.

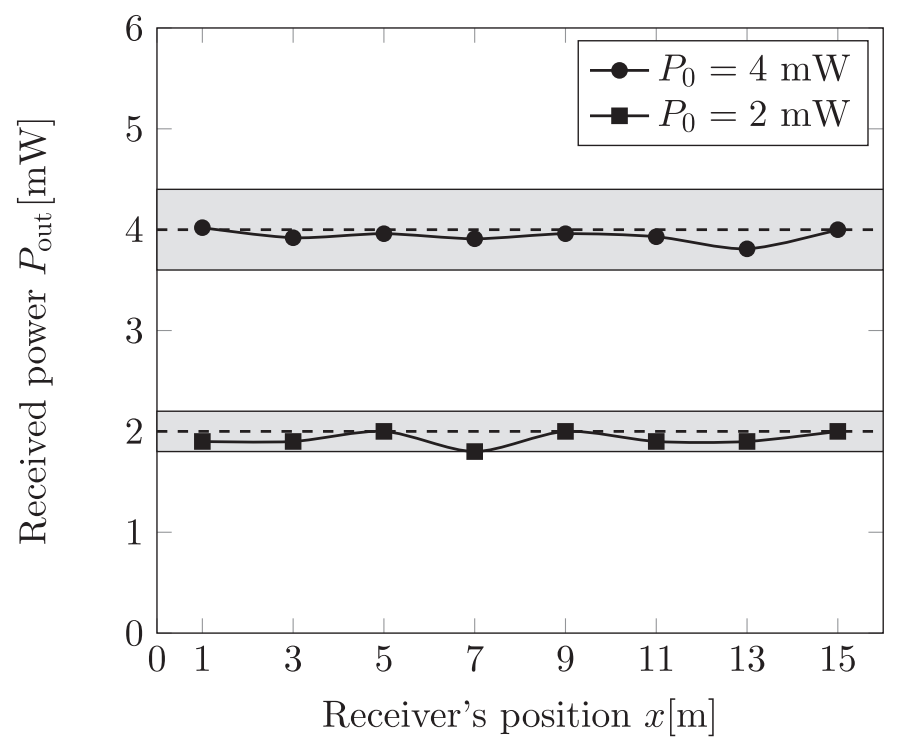

Fig. 8. Power profile when receiver-side load control is applied.

\subsection{Convergence}

Number of iterations for the load control in section 4.2 is provided in Fig. 9. It is shown in the figure that, the load control ends in, at most, 5 iterations. In the load control process, the most time consuming step is the measurement of load voltage which may last a duration from $1 \mathrm{~ms}$ to $10 \mathrm{~ms}$. Thus, the load control may last from about $5 \mathrm{~ms}$ to about $50 \mathrm{~ms}$. In the worst case when the load control lasts about $50 \mathrm{~ms}$, a vehicle with speed of $20 \mathrm{~km} / \mathrm{h}$ travels a distance of only about $0.3 \mathrm{~m}$, which is too short compared to $10 \%$ of the wavelength (about $1.6 \mathrm{~m}$ ) to affect the control process. In the best case when the load control lasts about $5 \mathrm{~ms}$, it is fast enough to keep pace with a vehicle moving at speed of $200 \mathrm{~km} / \mathrm{h}$. Indeed, duration of the load control can be further shortened by raising the tolerance $\varepsilon$. Therefore, convergence of the load control is promising to keep pace with an ordinary vehicle moving at speed of up to $100 \mathrm{~km} / \mathrm{h}$. 


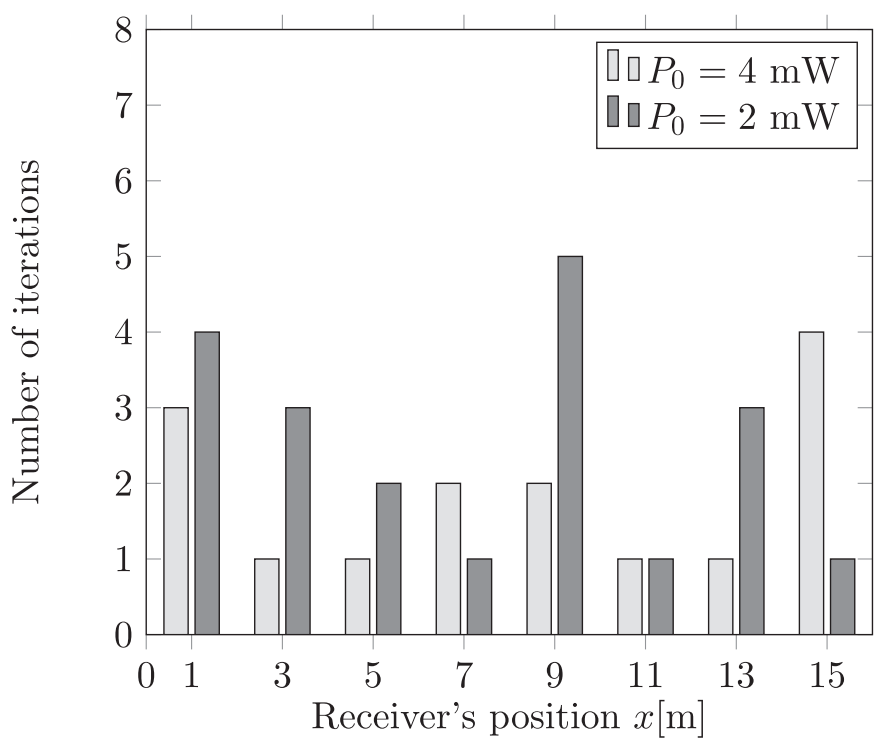

Fig. 9. Number of iterations for load control with tolerance $\varepsilon=0.1$.

\section{Conclusion}

We proposed a dynamic charging using hybrid inductively-capacitively coupled parallel line feeder with a receiver-side load control. Experimental results showed that the system is effective in guaranteeing a stable target power profile even under the impact of standing waves and receiver mobility. Analysis and experimental results also showed that the load control process is notification-free and fast converging compared to ordinary vehicle speed. Due to its low cost, compact size, simple structure and capability of on-demand power transfer, the proposed system is a practical option for dynamic charging of AGV and future EV.

\section{Acknowledgments}

This work was financially supported by DAIHEN Corporation. 